\title{
Strategy and Route-Planning Research for China's Engineering Science and Technology Development
}

\author{
Task Force for the Research on China's Engineering Science and Technology Development Strategy 2035 Comprehensive \\ Research Group
}

\begin{abstract}
Engineering science and technology is strongly linked with production, and is the most direct driving force for accomplishing the Two Centenary Goals of China. The main mission of strategic medium- and long-term engineering science and technology research is to develop a path to focus China's engineering science and technology toward scientific and technological power based on analyses of China's situation and development requirements. This paper discusses the methodology applied in our study for developing medium- and long-term engineering science and technology development strategies and proposes a general concept and strategic framework for China's engineering science and technology development through 2035.
\end{abstract}

Keywords: 2035; China's engineering science and technology development; strategic framework; route planning; strategic research method; technology foresight; requirement analysis

\section{Introduction}

Engineering science and technology is the key to creating real productivity because it has the closest connection with the society's economy, has the most immediate impacts to the society's economy, and has the most significant effects on the society's economy. Since the 12th Five-year Plan, China has made significant progress because of the continued implementation of its innovation-driven development strategy. In terms of scientific and technological innovation, the country has entered a new phase, where it is a follower in some fields, a parallel runner in other fields, and a frontrunner in still other fields. The central government has set ambitious targets during this new historical period, that is, the country is expected to join the ranks of innovative countries by 2020 , become one of the leading innovative countries by 2030 , and be a global powerhouse of science and technology by 2049 .

During the journey toward realizing China's vision of great transformation, engineering science and technology will need to meet huge demands for innovation and for putting a wide range of accomplishments into practice. In such a context, the Chinese Academy of Engineering (CAE) and the National Natural Science Foundation of China (NSFC) jointly launched a major advisory project entitled "Research on China's Engineering Science and Technology Development Strategy 2035" in 2015 based on the "Research on Medium- and Long-term Development Strategy of China's Engineering Science and Technology," [1] which was initiated in 2009. This project aimed to concentrate on the brilliant ideas of domestic and foreign academicians, experts, and personages from all social quarters; to develop a practical understanding of the trends of scientific advancement both home and abroad; and through envisioning the country's socioeconomic landscape in 2035, to identify major national strategic requirements. Based on such insights, the project will identify the trending areas in the fundamental research fields, for which proactive strategic planning is essential for the future development of engineering science and technology. In this way, China can fully leverage medium- and long-term scientific and technological route planning to overcome bottlenecks in the development of engineering science and technology, become

Received date: 25 December 2016; revised date:30 December 2016

Corresponding author: Wang Kunsheng. E-mail: wangks@spacechina.com

Funding program: CAE Advisory Project “Research on China's Engineering Science and Technology Development Strategy 2035" (2015-ZD-14); National Natural Science Foundation Project of China "Comprehensive Research on Development Strategy of China's Engineering Science and Technology towards 2035" (NSFC-L1524023)

Chinese version: Strategic Study of CAE 2017, 19 (1): 027-033

Cited item: Task Force for the Research on China's Engineering Science and Technology Development Strategy 2035 Comprehensive Research Group. Strategy and Route-Planning Research for China's Engineering Science and Technology Development. Strategic Study of CAE, https://doi.org/10.15302/J-SSCAE-2017.01.005 
a frontrunner in major engineering fields, improve its overall engineering strength, move faster toward meeting its goal of becoming a country strong in science and technology, and lay a solid foundation for a sustained long-term socioeconomic development.

\section{Methodology and research process for developing China's 2035 engineering science and technology development strategy}

\subsection{Research requirements}

This project aims to plan for the efficient development of engineering science and technology in China over the next 20 years; hence, its focus is on adopting an overarching strategic perspective, looking at an extensive range of issues of systemic importance, and adopting a long-term vision for the future. These research requirements are explicitly stated through four attributes. First, research should focus on future development and independent innovation, highlight the priority fields and core technologies expected to shape the future, and pay direct attention to potentially disruptive scientific discoveries and cutting-edge technologies. Second, the project should enhance top-level design and interdisciplinary research, use strategic and systemic thinking, study the key issues affecting national competitiveness and security, and consider the cross-disciplinary integration of science and technology. Third, to set the strategic priorities, efforts should be made to combine scientific and technological insights with socioeconomic research, conduct a multi-dimensional analysis of the development of engineering science and technology against the backdrop of an immense socioeconomic system, and remove current restrictions. Fourth, innovative research methodologies must be developed; organizational coordination must be enhanced; tasks must be delivered in a more scientific way that is in line with relevant standards; the importance of the overall planning and design of the methodology must be emphasized; workflows must be clearly defined; efficient expert teams must be developed; process control and coordination must be strengthened; and research efficiency and quality must be improved.

\subsection{Methodological system}

Based on domestic and international methods, theories, and practices for strategic science and technology research and combined with the features of engineering science and technology, a simple, relevant, and effective methodology is presented to ensure forward-looking scientific research that meets applicable standards (Fig. 1). The system consists of the following attributes:

(1) For conducting forward-looking research to develop medium- and long-term engineering science and technology strategies, quantitative approaches, such as bibliometrics and patent analysis, are combined with investigations by and discussions with experts. These activities will be conducted with a vision toward 2035 because foresight underpins the development of medium- and long-term engineering science and technology development strategies.

Technological foresight is a step-by-step exploration undertaken for the long-term planning of science, technology, economy, environment, and society. It stresses the importance of identifying key technologies and taking the initiative in shaping and creating the future. We can comprehend engineering science and technology development trends and achieve fundamental

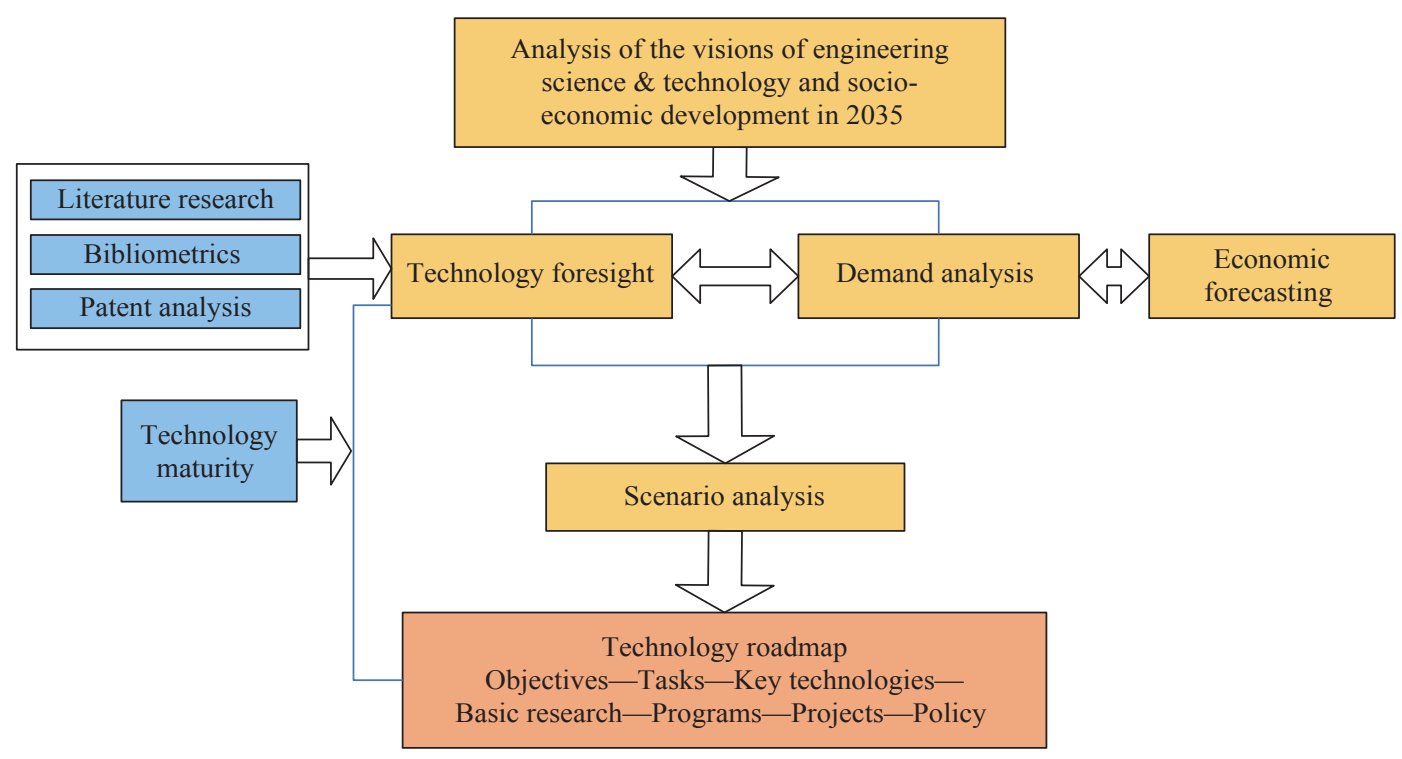

Fig. 1. Research project methodology. 
consensus on the selection of key technologies through studies of and exchanges between experts from various sectors. We can then use the concept of technological readiness to predict China's capabilities in various engineering science and technology fields by 2035 and develop timeframes for the realization of key technologies. Key technological projects and major technology sectors will be identified based on these insights from academicians and experts.

(2) The system should reflect the close connections between engineering science and technology and socioeconomic development, stress the role of demand in guiding the vision, and combine technological foresight with socioeconomic forecasts and demand analyses to guide the vision of future development and analyze possible scenarios.

The project has developed a "dynamic computable general equilibrium model of China's economy, energy, environment, and taxation (CN3ET-DCGE)" based on strategic studies and assessments made in various fields of engineering science and technology. This model showcases the changing factors guiding engineering science and technology technical progress and future energy sources and predicts China's societal, economic, energy, and environmental development by 2035. The project solicited opinions about China's 2035 socioeconomic outlook from all social groups using interviews of and questionnaires completed by experts to develop a list of socioeconomic development demands. It then used input from relevant engineering science and technology fields about these demands to define the requirements and promote the necessary developments in the related engineering domains.

(3) The system integrates an in-depth application of a technological roadmap with technological foresight and other methods of demand analysis. It develops a technological roadmap at the national strategic level that makes overarching assessments on the future of science, technology, the economy, society, and the environment. Engineering science and technology tasks can be identified and assigned at different stages based on this roadmap.

The technological roadmap generally covers the whole process from demand analysis to policy recommendations. This process is not a separate process but, rather, a part of the strategic research and combines technological foresight with demand analysis. It is not only a means of strategic research, but also one of its outputs. During the study, the results of the technological foresight and demand analysis feed the analyses of technological readiness from basic research and key technological R\&D to industrial applications. The inter-relationships between and among the demands, objectives, major tasks, choices of key technologies, basic research projects, major programs, key projects, and policy analysis are identified through correlation and optimized analysis to derive the engineering science and technology development path.

(4) The future directions of basic through strategic research must be identified to guide China's continued development of engineering science and technology. Importance is given to the balance between national demands and the anticipated feasibility of developing such engineering science and technology. Starting from general knowledge about the laws governing engineering, efforts should be made to summarize the key issues affecting the development of engineering science and technology.

In line with the future directions of major technologies, the basic research areas that underpin future engineering science and technology development should be identified by field-specific task forces based on selection criteria, such as cutting-edge R\&D with challenges, general demands, technical bottlenecks and breakthroughs, and applications in key engineering projects. On this basis, a literature review and an analysis of the landscape of basic research are performed before developing suggestions about the subjects, content, and order of priorities for the basic research fields that require greater attention from policymakers to enable further progress.

(5) Having the full participation of high-end national thinktanks, such as the CAE and the NSFC, which perform strategic studies and develop technological foresight, is essential. Engaging a wide range of academicians and experts in the field of social sciences during the brainstorming process is also necessary. At the same time, efforts should be made to solicit opinions and suggestions from international engineering science and technology experts through international forums and other channels to enhance the scientific nature of the strategic research.

\subsection{Research process}

According to the requirements of strengthening the overarching research and top-level design and combining with all relevant research methods, this project proposes a strategic research process with three stages: general description, breakdown into fields, and summary. In the first phase of the "general description," efforts should be made to improve the overall design. Guided by the overall plan, the general description presents a complete picture of the various specific fields, and an integrated study is conducted based on the combination of technological foresight, demand analysis, and economic forecast. Research on strategic thinking in engineering fields is concurrently conducted. During the second phase, the focus is on each engineering field and its corresponding task force. An in-depth study is conducted in each field based on the previous general research, keeping the whole picture in mind. The outputs include the development of priorities and roadmaps. During the third phase of the "summary," efforts should be made based on the in-depth studies of the preceding phase to identify the overall strategic focus and any engineering issues, conduct discussions about special topics, make research-based judgments, and integrate the research findings. At this stage, discussions regarding interdisciplinary development are of particular importance. The aim is to develop an overall strategy and path for the development of China's engineering science and technology through 2035. 
In summary, the project begins with an overarching research, followed by in-depth studies in the subsidiary fields. The entire process highlights the combination of the overall vision with specific tasks, integrates the parts into a whole, and summarizes the key findings and their interrelations. Such a process enables the research efforts to be more comprehensive, systematic, and scientific and ensures the effective management of the research process and the efficient integration of the research outcomes.

\section{Future trends and demands for engineering science and technology development}

\subsection{Prospects of engineering science and technology on the global scale by 2035}

At present, the world has entered the age of intensive innovation and industrial revolutions. Countries around the world are all accelerating efforts to plan for future scientific and technological development. Moreover, innovations in technology and modalities in various fields are continually emerging. As 2035 approaches, worldwide engineering science and technology development is expected to dramatically change economies, societies, and lifestyles.

\subsubsection{An intelligent era of the "Internet of Things" will arrive by} 2035

Mankind will be coexisting with an intelligent network that will connect everything, enjoying mobile, ubiquitous, and intelligent network services based on ultra-wide bandwidth and low latency. Advanced interaction and display technologies will have created seamless connections between real and digital worlds, wherein one can enjoy highly simulated situations despite long distances. Quantum, brain-like, biological, and optical computing will overturn traditional computing models. Intelligent software, which can perceive, learn, evolve, and collaborate, will have become ubiquitous. Wearable devices, robots, and intelligent control systems will have greatly improved production and upgraded social life to a more intelligent level, where manmachine integration has become a reality.

3.1.2 A brand-new energy system that is low in carbon, green, efficient, intelligent, and network-based will have been gradually established

Over the next 20 years, a revolution in energy technologies will bring profound changes to the global landscape of energy production and consumption. The share of renewable energy in the global energy supply is expected to rise to approximately $31 \%$ [2], which will promote the gradual expansion of intelligently distributed energy services. By 2035, the energy sector and electric power industry will have become an integrated energy system. Global energy and mining systems will have developed along with ecological, technological, and socioeconomic systems. The world will have struck a balance among energy conversion, storage, sharing, and application.

3.1.3 An intelligent, sustainable, and flexible manufacturing industry will have been established

Based on the concept of green development and the extensive application of the network information technology, revolutionary changes are expected to occur in the morphology of factories, as well as in the modalities of the manufacturing industries' production and service. Additive, biological, and micro- and nanoscale manufacturing will have been extensively applied. Intelligent production modes will gradually become more popular. The rigid way, in which the manufacturing sector's production is organized, will have shifted toward being highly flexible and intelligent. Concentrated mass manufacturing will coexist with distributed collaborative manufacturing. The manufacturing service sector will also have made a significant headway. The linkages of the industrial ecology will become very far-reaching. Clean and self-sustaining manufacturing processes and modalities with low consumption levels will have been developed. The methods of production for products and services will become digital, network-based, intelligent, personalized, and highly flexible.

3.1.4 Future cities will become eco-friendly, livable, intelligent, and safe

In 2035, cities and urban layouts and urban system planning will pay more attention to the philosophies of putting humans first and valuing ecological harmony. Urban development and its operational patterns will appropriately balance technology and the humanities, traditions and modernization, and life and ecological protection. Urban construction, transportation, and infrastructure, such as pipelines and networks, will become threedimensional, network-based, and intelligent. Transportation within and between cities will be smooth, fast, and intelligent. Cities will be managed in a smoother and more efficient manner through implementing sensible, controllable, and highly intelligent management approaches; will be more resilient to risks; and will be more capable of safeguarding the overall public security. Thus, cities will become holistic and strike a good balance between their various functions based on sound urban structures. Such cities will be able to support reasonable loads, offer a good environment, and be open and self-sustaining. They will present characteristics of "ecosystems" that run in a balanced and orderly manner, are intelligent, and can adjust whenever necessary.

3.1.5 Wide area networking and digital inclusion services will gradually make the highly integrated and efficient blue economy integral to the economy

By 2035, space exploration and utilization will have entered a new era. Communication, navigation, remote sensing satellites, and ground information systems will have been widely interconnected, thereby forming an integrated information network of the 
"sky, space, ground, and sea." Such a network will provide accurate, real-time, ubiquitous, and integrated spatial information services. Space travel over longer distances will have become cheaper and more reliable. Manned probes to the moon and nearEarth space tours will gradually come into fashion. Meanwhile, ocean exploration, development, utilization, and protection will have become hot topics globally. By 2035, the world will be capable of monitoring and analyzing oceanic environments at the macro-scale globally and at the micro-scale regionally. Ocean monitoring and exploration that attempt to reach the "deep sea afar" will play an increasingly active role. Undersea mines, offshore factories, and sea cities will be booming. A "blue industrial civilization" is expected to take shape, mainly featuring efforts to explore strategic marine resources.

3.1.6 A healthy lifestyle that enables man to live in harmony with nature will gradually be developed

With the goal of "everybody enjoys health," advancing science and technology, changing unsuitable modalities, improving living environments and medical capabilities, substantially upgrading nutrition and health levels, and improving disease control and prevention capabilities are necessary. By 2035, the development and application of biotechnology will have made agricultural and food production more efficient, greener, and safer. Countries will eventually turn to across-the-board agricultural modernization that promotes mechanization, quality seeds, electrification, IT applications, and environmental protection. Emerging industrial farms are expected to greatly enhance the efficiency of agricultural production and provide ecological benefits. Proactive pollution control systems will have a profound role in food production and in everyday life. Global environmental issues will have been further controlled and addressed. The degree to which environmental pollution affects health is expected to decline to the lowest level in modern times. Chronic and infectious diseases will have been effectively prevented and controlled. Tissue reengineering will no longer be difficult. Major breakthroughs will have been made in modern pharmaceutical technologies. Medical treatments will gradually become noninvasive, precise, intelligent, and accessible, even over long distances. Precision medical systems will be promoted. Global average life expectancy will be further increased.

\subsection{Vision of China's socioeconomic development toward 2035 and demands for engineering science and technology development}

China is committed to meeting the objective of becoming a world-class country, strong in science and technology, and undertaking the great rejuvenation of the Chinese nation; hence, we envision that by 2035, the following developments will have taken place: China will be highly globalized, and will have become the largest economy in the world and the most powerful engine of world economic growth. It will be engaging in global affairs to an unprecedented depth and breadth with substantial international clout.

By 2035, China is expected to enter the rank of high-income countries. Driven by innovation, its economy will be steadily and rapidly growing. Consumption will have become the primary driving force for development. Its industrial structure will have become more rational. Diverse and individualized consumer demands will be increasingly gratified.

In 2035, China will have become an intelligent country. It will have reached a world-class level of advanced connectivity. Its new intelligent manufacturing system will be fully functional and rank among the bests in the world. Intelligent cities, transportation systems, medical systems, and education will all be parts of people's lives.

In 2035, China will be a country of sustainable development. A new clean, efficient, intelligent, and interconnected energy system will have taken shape. A sustainable national economic system that includes recycling and quantitative reduction will have been established. The ecological environment will have been notably improved. The public will have adopted a green lifestyle, and China will enter a new era of ecological civilization.

In 2035, China will be a harmonious country. It will have met its goal of being governed by the rule of law. Citizens will enjoy a more rational and equitable access to health care and a more equitable access to higher-quality education resources.

In 2035, China will be more secure. It will have developed strong capabilities of preventing and mitigating natural hazards with a three-dimensional public security network that covers the full range of issues. It will be equipped with capabilities to proactively defend public security. It will also have significantly improved its capabilities to upgrade people's living standards and safeguard public security.

China is faced with the constant, rapid advancement of science and technology across the globe; therefore, planning for China's engineering science and technology development based on an objective analysis of the current situation with the goals of developing new dimensions in China's society and economy to support the vision of 2035 is essential. At present, China has substantially enhanced its overall performance in engineering science and technology because of its previous long-term efforts. The country is at an important transition toward qualitative leaps from quantitative accumulation and toward an upgrade of systemic capabilities from making incremental breakthroughs. It has already become a world leader in certain fields and in areas of engineering science and technology. Nevertheless, the country's foundation of science and technology innovation requires further enhancement. More professional teams and resources are required in various fields of engineering science and technology to enhance capacity. More momentum should be provided to support the originality that underpins homegrown innovations. 
Fundamental changes have not yet taken place in some key areas, such as electronic information, high-end and intelligent equipment, high-end instruments, core components and key raw materials, new drugs, and medical engineering, and the country remains dependent on imported core technologies. Thus, the development of corresponding industries has been restricted, which in turn, leads to a weak industrial foundation and insufficient capabilities. In addition, the country has been affected in fields, such as energy, resources, environment, and ecology, by a combination of factors, including standards, norms, economic patterns, and industrial ecology. More efforts should be made to enhance the capability of making integrated innovations through collaboration across different fields and disciplines. Based on the results of engineering science and technology foresight and analyses, in more than 800 proposed important technological areas over the next 20 years, notable gaps exist between China and the world's leading countries in terms of the current R\&D ability and international competitiveness. The country is in a relatively regressive position in about a quarter of these key technological areas. These facts highlight the still-arduous task of making innovations in engineering science and technology and enabling a leapfrog development in China.

\section{Strategic thinking on China's engineering science and technology toward 2035}

\subsection{Ideas for development}

The development of China's engineering science and technology over the next 20 years should focus on meeting major national strategic demands and realizing the concepts of innovation, coordination, greenness, openness, and sharing. With a systemic understanding of engineering science and technology laws and trends, it is important to plan well in advance by having a holistic and innovative plan for basic research, technological R\&D, and engineering applications. With major engineering projects as driving forces and with support from scientific breakthroughs, China should accelerate its efforts to develop homegrown, advanced, and applicable engineering technologies; should promote digitization, connectivity, intelligence, greenness, and precision; and should provide better services in all fields. In engineering fields, where China currently boasts strength, efforts should be made to advance the country to the lead.

The following principles should be underlined during the engineering science and technology development: first, we must anticipate and plan, blaze the trail for a strong and innovative country, and recognize that taking a commanding lead in science and technology is in the overall long-term interest; second, we must remove bottlenecks, facilitate independent and coordinated development, and change the situation depending on other countries' core technologies in key fields as soon as possible; third, we need to plan for the development of technology clusters, enable them to collaborate and make progress across the board, and strengthen the coordinated development and integrated application of technologies in various fields from the perspective of an overall system; and fourth, we need to strengthen open collaboration, optimize the ecological network of innovation, and move China up in the global innovation rankings.

\subsection{Strategic thinking and objectives}

The ultimate mission of engineering science and technology is to promote socioeconomic development, safeguard a living-friendly environment, and elevate people's living standards. In line with China's vision of socioeconomic development and the corresponding demands for engineering science and technology over the next 20 years, the engineering sector should be developed from the starting point of putting people first and be headed in the main direction of being intelligent, green, and sustainable. Efforts should be made to take a commanding lead in new technological revolutions, develop advantages and capabilities in key strategic areas, and vigorously deliver the strategy of "Made in China 2025" by focusing on the development of new systems for energy, industry, and people's livelihoods. We must expedite efforts to build a country strong in connectivity, sea exploration, and space investigation while sparing no efforts to develop a beautiful, healthy, and safe China. To this end, an overall framework for developing engineering science and technology through 2035 is proposed (Fig. 2) from the six aspects of information infrastructure, energy security, industrial upgrades, modern cities, space and ocean, and health.

4.2.1 Build a strong information base to take the lead in the intelligent era

In 2035, the human society will have entered an information age generally characterized by intelligence. Information technology, with its in-depth integration with physical, industrial, and social systems, and all aspects of people's lives, is expected to lead and drive overall economic and social progress. Therefore, one of the most important foundations for building a country strong in science and technology is the across-the-board leapfrog development of the electronic information technology. Toward 2035 , vigorously strengthening basic research on new concepts, systems, and materials among other cutting-edge issues in electronic information will be important tasks for engineering science and technology. Efforts should be made to accelerate the removal of bottlenecks in the chip and integrated circuit (IC) technologies and basic and core software. Taking the lead in making breakthroughs in high-performance computing technology, intelligent technology, space-air-ground integration of network-based information technology, next generation Internet, and advanced interaction and display technologies, among other cutting-edge areas, is necessary. We should focus on advancing 


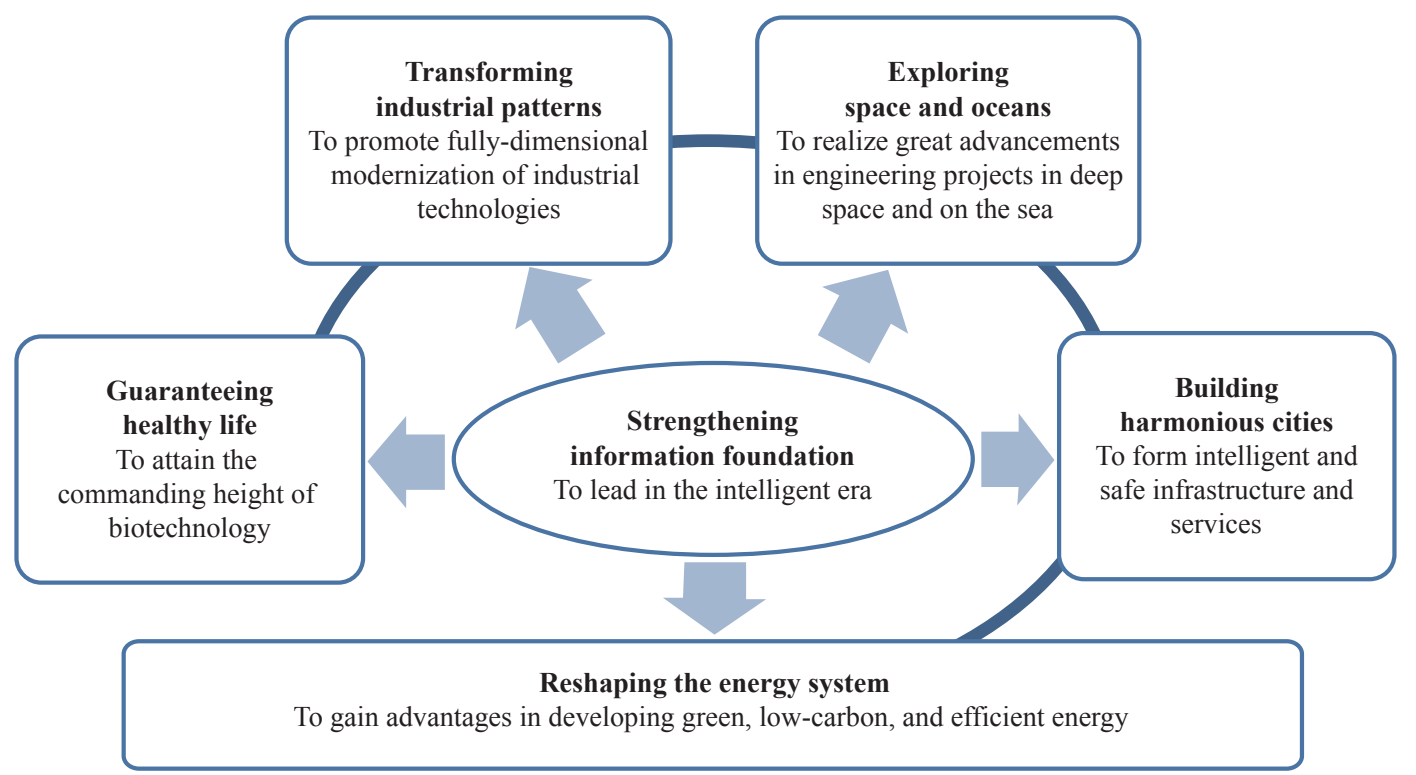

Fig. 2. Framework for China's development of engineering science and technology through 2035.

version 2.0 of artificial intelligence and vigorously promote the comprehensive application of advanced information technology.

\subsubsection{Industrial patterns should be transformed and upgraded} to promote a fully dimensional modernization of industrial technologies

Considering the future scenario, wherein industries will all become intelligent, network-based, green, and service-oriented, generic industrial technologies focusing on nanometer materials, composite materials, nano manufacturing at the micro scale, additive manufacturing, biological manufacturing, robots, and other areas of advanced materials and manufacturing technologies must be vigorously developed. We must actively enable the entire system of factories and industries to become intelligent and develop a manufacturing modality that is clean and self-sustaining with low consumption based on the green development philosophy. We must link industries, society, and the ecological system together, and realize an economical, efficient, and clean production.

4.2.3 Reshaping the energy system and creating advantages in developing green, low-carbon, and efficient energy are necessary

Being competitive in energy technology is strategically imperative because countries seek to meet future energy demands and sustain future socioeconomic growth and efficient green development. Considering 2035, the R\&D of technologies that save energy and reduce emissions must be prioritized in line with the objective laws governing the development of energy and resources. While being committed to the technological R\&D for the clean utilization of traditional energies, efforts should also be made to focus on the technological R\&D of the unconventional exploitation and utilization of fossil fuels, efficient use of renewable energies, and advanced intelligent distribution-type networks of integrated energy sources. Scientific and technological (S\&T) innovations in green supply-side development must be expedited, and the consumption side must use S\&T innovations in a clean, low-carbon, and concentrated manner. We must promote a revolution to reduce the use of high-carbon energy, increase the use of clean energy, and improve the efficiency of energy systems. The goals are to gain advantages in the competition for energy technologies and industrial development, ensure the delivery of national socioeconomic development strategies, and take the initiative of development under the future global framework of energy governance.

4.2.4 Building harmonious cities with intelligent and safe infrastructure and services is important

The goal is to lead the innovative development of new urbanization, adapt to the transformative changes in the patterns of regional coordinated development, and accomplish ecological and green development. We must develop theories, methods, and technological systems for eco-cities and green buildings, which are consistent with the situation of China and the international trend, based on the development philosophies of regarding people as the most important, stressing efficient coordination, and balancing the development of the humanities, sciences, technology, and ecology. We must also develop urban and transportation infrastructure and integrated transportation systems that are advanced, safe, reliable, intelligent, and healthy. Furthermore, we must build systems to develop and utilize water resources and improve coordinated operation, service quality, and capability to proactively guarantee the safety of various transportation modes. Efforts should also be made to develop urban management technologies and operational systems that augment highly intelligent 
management and intelligent services to guarantee harmonious urban development and sound urban systems in the future.

4.2.5 Exploring space and oceans is important in realizing great advancements in engineering projects

We support development strategies for building a country strong in aerospace and ocean technologies; working at the frontiers of deep space and sea exploration and utilization; aiming to meet future demands for precise, real-time, seamless, and ubiquitous spatial information services; and seeking to develop and utilize strategic ocean resources, secure marine safety and related rights and interests, and protect the ocean environment. In addition to these efforts, we shall give full due to the role of the market and businesses as key agents of innovation while fully leveraging the driving forces provided by national strategies and the systemic advantage of effective resource mobilization to accomplish large projects. We must pool all our efforts to conduct R\&D in key space and ocean exploration engineering technologies. Efforts should also be made to overtake competitors in key ocean and space exploration technologies and enable leapfrog development, which is imperative to fostering space and ocean economies as important future pillars.

4.2.6 Promoting healthy development by building comprehensive science and technology systems that cover every aspect of major processes is important

A healthy life is the basic goal of mankind and, therefore, a persistent pursuit. A healthy environment guarantees healthy living and sound development. With healthy development as the ultimate goal, we must vigorously promote the R\&D and application of information technology and biotechnology and improve the outlook for agriculture, medicine, and health care. With food safety and agricultural eco-security as the hub, we must establish an innovative agricultural engineering science and technology system in accordance with China's status as a major agricultural country. This system must be up to world-class standards and must be intelligent, efficient, ecology-friendly, green, and safe. As regards pollution prevention and control, the role of scientific and technological innovation should be fully leveraged throughout the process: reducing pollution at its sources, process control, and recycling. Technological systems that monitor multiple pollutants on a cross-regional basis should be set up to bolster all-around pollution prevention and control, environmental improvement, and ecological restoration. Guided by modern concepts of medical care, we must vigorously promote biotechnology, brain-like science, tissue engineering and organ regeneration technologies, the R\&D of modern drugs, high-end medical instruments, intelligent equipment, and modernized traditional Chinese medicine. Efforts should be made to develop a modern, world-class health care system. We must advance the overall medical development in China, including the explanations of disease and health mechanisms, prevention and intervention, precision medicine, and holistic integrated medicine.

Based on the abovementioned framework and China's national conditions, this project puts forward the ideas, key tasks, directions for key technologies, and development roadmaps for 12 engineering fields, including energy and resources, environment and ecology, information and electronics, advanced materials, equipment manufacturing, process manufacturing, urbanization and infrastructure, transportation, space and ocean, modern agriculture, medicine and health, and public safety. It also presents major engineering projects, major R\&D projects in engineering, and basic research areas that require early planning to pave the way for future engineering technology and advance toward 2035.

Overall, based on a national strategic deployment, by 2030 , China will have become one of the leading innovative countries through its hard work; its driving force for development should have been fundamentally changed; its socioeconomic development should have reached a much higher level; and its international competitiveness should be significantly improved [3]. Based on the improvements in all fronts, by 2035, further efforts will have been made to consolidate the sound foundation of universal technologies, thereby substantially enhancing China's own innovation capabilities and giving priority to the development of engineering science and technology. Industrial technology systems that are internationally competitive, green, clean, safe, and controllable must be established along with a full-fledged eco-system and a sound technological system for a healthy China. We should seek to make original accomplishments to the substantial benefit of the world's engineering science and technology and the progress of human civilization and, thus, stimulate an innovation-driven socioeconomic development across the board.

\section{Conclusions}

This study of the medium- and long-term development strategies for engineering science and technology touches on a broad range of issues, adopts a forward-looking perspective, and needs to be highly strategic, scientific, and rational. In performing the research, the project team has developed a deep understanding of this task's complexity and difficulty. During the two-year project cycle, we had almost simultaneously conducted technological foresight, demand analysis, and strategic studies of various fields and created a technological roadmap. There is still room for improvement in terms of method application and the breadth and depth of strategic studies. At the same time, the environment, in which engineering technology develops, is constantly changing. It follows that such research is a long-term endeavor that calls for an ever-increasing depth of understanding and continuous verification. 
For these reasons, the NSFC and the CAE have decided to jointly conduct a research on the medium- and long-term development strategies for engineering science and technology as a long-term task based on a rolling five-year cycle. Direct attention must be given not only to the top-level design of the project, but also to the strengthening of the systemic design and organized collaboration within the five-year research cycle and over longer terms. Highlighting the core mission of drafting macro and micro engineering development strategies over the next 20 years and connecting the dots of demand analysis, technology foresight, strategic studies, and technological roadmaps in key fields are important. Some of these will be performed in parallel to facilitate better correlations, enable mutual progress, and take gradual steps to ensure a systematic, progressive, and scientific strategic research process based on lessons learned and the accumulation of new knowledge. Through a combination of the macro strategic study and the micro technological roadmap, we seek to provide more forward-looking research outcomes to deliver more effective services to enhance macro decision-making and S\&T policy making at the national level and substantially contribute to China's pursuit of being a country strong in science and technology.

\section{References}

[1] Project team of Research on Medium- and Long-Term Development Strategy of China's Engineering Science and Technology. Research on medium and long-term development strategy of China's engineering science and technology $[\mathrm{M}]$. Beijing: China Science \& Technology Press, 2015. Chinese.

[2] International Energy Agency. World energy development report [EB/OL]. (2016-08-20) [2016-12-15]. http://finance.sina.com.cn roll/2016-11-07/doc-ifxxmyuk6086724.shtml. Chinese.

[3] State Council of the People's Republic of China. Outline of the national strategy of innovation-driven development [EB/OL]. (201605-20) [2016-09-20]. http://www.scio.gov.cn/xwfbh/xwbfbh/ wqfbh/33978/34585/xgzc34591/Document/1478339/1478339_1. htm. Chinese. 